\title{
Boundedness of localization operators on Lorentz mixed-normed modulation spaces
}

\author{
Ayşe Sandıkç ${ }^{*}$ \\ Dedicated to Professor Ravi P Agarwal
}

\author{
"Correspondence: \\ ayses@omu.edu.tr \\ Department of Mathematics, \\ Faculty of Arts and Sciences, \\ Ondokuz Mayıs University, Samsun, \\ Turkey
}

\begin{abstract}
In this work we study certain boundedness properties for localization operators on Lorentz mixed-normed modulation spaces, when the operator symbols belong to appropriate modulation spaces, Wiener amalgam spaces, and Lorentz spaces with mixed norms.
\end{abstract}

Keywords: localization operator; Lorentz spaces; Lorentz mixed normed spaces; Lorentz mixed-normed modulation spaces; Wiener amalgam spaces

\section{Introduction}

In this paper we will work on $\mathbb{R}^{d}$ with Lebesgue measure $d x$. We denote by $\mathcal{S}\left(\mathbb{R}^{d}\right)$ the space of complex-valued continuous functions on $\mathbb{R}^{d}$ rapidly decreasing at infinity. For any function $f: \mathbb{R}^{d} \rightarrow \mathbb{C}$, the translation and modulation operator are defined as $T_{x} f(t)=$ $f(t-x)$ and $M_{w} f(t)=e^{2 \pi i w t} f(t)$ for $x, w \in \mathbb{R}^{d}$, respectively. For $1 \leq p \leq \infty$, we write the Lebesgue spaces $\left(L^{p}\left(\mathbb{R}^{d}\right),\|\cdot\|_{p}\right)$.

Let $\langle x, t\rangle=\sum_{i=1}^{d} x_{i} t_{i}$ be the usual scalar product on $\mathbb{R}^{d}$. The Fourier transform $\hat{f}$ (or $\mathcal{F} f$ ) of $f \in L^{1}\left(\mathbb{R}^{d}\right)$ is defined to be

$$
\hat{f}(t)=\int_{\mathbb{R}^{d}} f(x) e^{-2 \pi i\langle x, t\rangle} d x
$$

For a fixed nonzero $g \in \mathcal{S}\left(\mathbb{R}^{d}\right)$ the short-time Fourier transform (STFT) of a function $f \in \mathcal{S}^{\prime}\left(\mathbb{R}^{d}\right)$ with respect to the window $g$ is defined as

$$
V_{g} f(x, w)=\left\langle f, M_{w} T_{x} g\right\rangle=\int_{\mathbb{R}^{d}} f(t) \overline{g(t-x)} e^{-2 \pi i t w} d t,
$$

for $x, w \in \mathbb{R}^{d}$. Then the localization operator $A_{a}^{\varphi_{1}, \varphi_{2}}$ with symbol $a$ and windows $\varphi_{1}, \varphi_{2}$ is defined to be

$$
A_{a}^{\varphi_{1}, \varphi_{2}} f(t)=\int_{\mathbb{R}^{2 d}} a(x, w) V_{\varphi_{1}} f(x, w) M_{w} T_{x} \varphi_{2} d x d w .
$$

O2014 Sandıkçi; licensee Springer. This is an Open Access article distributed under the terms of the Creative Commons Attribution License (http://creativecommons.org/licenses/by/2.0), which permits unrestricted use, distribution, and reproduction in any medium, provided the original work is properly cited. 
If $a \in \mathcal{S}^{\prime}\left(\mathbb{R}^{d}\right)$ and $\varphi_{1}, \varphi_{2} \in \mathcal{S}\left(\mathbb{R}^{d}\right)$, then the localization operator is a well-defined continuous operator from $\mathcal{S}\left(\mathbb{R}^{d}\right)$ to $\mathcal{S}^{\prime}\left(\mathbb{R}^{d}\right)$. Moreover, it is to be interpreted in a weak sense as

$$
\left\langle A_{a}^{\varphi_{1}, \varphi_{2}} f, g\right\rangle=\left\langle a V_{\varphi_{1}} f, V_{\varphi_{2}} g\right\rangle=\left\langle a, \overline{V_{\varphi_{1}} f} V_{\varphi_{2}} g\right\rangle
$$

for $f, g \in \mathcal{S}\left(\mathbb{R}^{d}\right),[1,2]$.

Fix a nonzero window $g \in \mathcal{S}\left(\mathbb{R}^{d}\right)$ and $1 \leq p, q \leq \infty$. Then the modulation space $M^{p, q}\left(\mathbb{R}^{d}\right)$ consists of all tempered distributions $f \in \mathcal{S}^{\prime}\left(\mathbb{R}^{d}\right)$ such that the short-time Fourier transform $V_{g} f$ is in the mixed-norm space $L^{p, q}\left(\mathbb{R}^{2 d}\right)$. The norm on $M^{p, q}\left(\mathbb{R}^{d}\right)$ is $\|f\|_{M^{p, q}}=$ $\left\|V_{g} f\right\|_{L^{p, q}}$. If $p=q$, then we write $M^{p}\left(\mathbb{R}^{d}\right)$ instead of $M^{p, p}\left(\mathbb{R}^{d}\right)$. Modulation spaces are Banach spaces whose definitions are independent of the choice of the window $g$ (see $[2,3]$ ).

$L(p, q)$ spaces are function spaces that are closely related to $L^{p}$ spaces. We consider complex-valued measurable functions $f$ defined on a measure space $(X, \mu)$. The measure $\mu$ is assumed to be nonnegative. We assume that the functions $f$ are finite valued a.e. and some $y>0, \mu\left(E_{y}\right)<\infty$, where $E_{y}=E_{y}[f]=\{x \in X|| f(x) \mid>y\}$. Then, for $y>0$,

$$
\lambda_{f}(y)=\mu\left(E_{y}\right)=\mu(\{x \in X|| f(x) \mid>y\})
$$

is the distribution function of $f$. The rearrangement of $f$ is given by

$$
f^{*}(t)=\inf \left\{y>0 \mid \lambda_{f}(y) \leq t\right\}=\sup \left\{y>0 \mid \lambda_{f}(y)>t\right\}
$$

for $t>0$. The average function of $f$ is also defined by

$$
f^{* *}(x)=\frac{1}{x} \int_{0}^{x} f^{*}(t) d t
$$

Note that $\lambda_{f}, f^{*}$, and $f^{* *}$ are nonincreasing and right continuous functions on $(0, \infty)$. If $\lambda_{f}(y)$ is continuous and strictly decreasing then $f^{*}(t)$ is the inverse function of $\lambda_{f}(y)$. The most important property of $f^{*}$ is that it has the same distribution function as $f$. It follows that

$$
\left(\int_{X}|f(x)|^{p} d \mu(x)\right)^{\frac{1}{p}}=\left(\int_{0}^{\infty}\left[f^{*}(t)\right]^{p} d t\right)^{\frac{1}{p}}
$$

The Lorentz space denoted by $L(p, q)(X, \mu)$ (shortly $L(p, q)$ ) is defined to be vector space of all (equivalence classes) of measurable functions $f$ such that $\|f\|_{p q}^{*}<\infty$, where

$$
\|f\|_{p q}^{*}= \begin{cases}\left(\frac{q}{p} \int_{0}^{\infty} t^{\frac{q}{p}-1}\left[f^{*}(t)\right]^{q} d t\right)^{\frac{1}{q},} & 0<p, q<\infty, \\ \sup _{t>0} t^{\frac{1}{p}} f^{*}(t), & 0<p \leq q=\infty .\end{cases}
$$

By (1.1), it follows that $\|f\|_{p p}^{*}=\|f\|_{p}$ and so $L(p, p)=L^{p}$. Also, $L(p, q)(X, \mu)$ is a normed space with the norm

$$
\|f\|_{p q}= \begin{cases}\left(\frac{q}{p} \int_{0}^{\infty} t^{\frac{q}{p}-1}\left[f^{* *}(t)\right]^{q} d t\right)^{\frac{1}{q}}, & 0<p, q<\infty, \\ \sup _{t>0} t^{\frac{1}{p}} f^{* *}(t), & 0<p \leq q=\infty .\end{cases}
$$


For any one of the cases $p=q=1 ; p=q=\infty$ or $1<p<\infty$ and $1 \leq q \leq \infty$, the Lorentz space $L(p, q)(X, \mu)$ is a Banach space with respect to the norm $\|\cdot\|_{p q}$. It is also well known that if $1<p<\infty, 1 \leq q \leq \infty$ we have

$$
\|\cdot\|_{p q}^{*} \leq\|\cdot\|_{p q} \leq \frac{p}{p-1}\|\cdot\|_{p q}^{*}
$$

(see $[4,5])$.

Let $X$ and $Y$ be two measure spaces with $\sigma$-finite measures $\mu$ and $\nu$, respectively, and let $f$ be a complex-valued measurable function on $(X \times Y, \mu \times v), 1<P=\left(p_{1}, p_{2}\right)<\infty$, and $1 \leq Q=\left(q_{1}, q_{2}\right) \leq \infty$. The Lorentz mixed norm space $L(P, Q)=L(P, Q)(X \times Y)$ is defined by

$$
L(P, Q)=L\left(p_{2}, q_{2}\right)\left[L\left(p_{1}, q_{1}\right)\right]=\left\{f:\|f\|_{P Q}=\|f\|_{L\left(p_{2}, q_{2}\right)\left(L\left(p_{1}, q_{1}\right)\right)}=\|\| f\left\|_{p_{1} q_{1}}\right\|_{p_{2} q_{2}}<\infty\right\} .
$$

Thus, $L(P, Q)$ occurs by taking an $L\left(p_{1}, q_{1}\right)$-norm with respect to the first variable and an $L\left(p_{2}, q_{2}\right)$-norm with respect to the second variable. The $L(P, Q)$ space is a Banach space under the norm $\|\cdot\|_{P Q}$ (see $\left.[6,7]\right)$.

Fix a window function $g \in \mathcal{S}\left(\mathbb{R}^{d}\right) \backslash\{0\}, 1 \leq P=\left(p_{1}, p_{2}\right)<\infty$, and $1 \leq Q=\left(q_{1}, q_{2}\right) \leq \infty$. We let $M(P, Q)\left(\mathbb{R}^{d}\right)$ denote the subspace of tempered distributions $\mathcal{S}^{\prime}\left(\mathbb{R}^{d}\right)$ consisting of $f \in \mathcal{S}^{\prime}\left(\mathbb{R}^{d}\right)$ such that the Gabor transform $V_{g} f$ of $f$ is in the Lorentz mixed norm space $L(P, Q)\left(\mathbb{R}^{2 d}\right)$. We endow it with the norm $\|f\|_{M(P, Q)}=\left\|V_{g} f\right\|_{P Q}$, where $\|\cdot\|_{P Q}$ is the norm of the Lorentz mixed norm space. It is well known that $M(P, Q)\left(\mathbb{R}^{d}\right)$ is a Banach space and different windows yield equivalent norms. If $p_{1}=q_{1}=p$ and $p_{2}=q_{2}=q$, then the space $M(P, Q)\left(\mathbb{R}^{d}\right)$ is the standard modulation space $M^{p, q}\left(\mathbb{R}^{d}\right)$, and if $P=p$ and $Q=q$, in this case $M(P, Q)\left(\mathbb{R}^{d}\right)=M(p, q)\left(\mathbb{R}^{d}\right)$ (see $[8,9]$ ), where the space $M(p, q)\left(\mathbb{R}^{d}\right)$ is Lorentz type modulation space (see [10]). Furthermore, the space $M(p, q)\left(\mathbb{R}^{d}\right)$ was generalized to $M(p, q, w)\left(\mathbb{R}^{d}\right)$ by taking weighted Lorentz space rather than Lorentz space (see $\left.[11,12]\right)$.

In this paper, we will denote the Lorentz space by $L(p, q)$, the Lorentz mixed norm space by $L(P, Q)$, the standard modulation space by $M^{p, q}$, the Lorentz type modulation space by $M(p, q)$, and the Lorentz mixed-normed modulation space by $M(P, Q)$.

Let $1 \leq r, s \leq \infty$. Fix a compact $Q \subset \mathbb{R}^{d}$ with nonempty interior. Then the Wiener amalgam space $W\left(L^{r}, L^{s}\right)\left(\mathbb{R}^{d}\right)$ with local component $L^{r}\left(\mathbb{R}^{d}\right)$ and global component $L^{s}\left(\mathbb{R}^{d}\right)$ is defined as the space of all measurable functions $f: \mathbb{R}^{d} \rightarrow \mathbb{C}$ such that $f \chi_{K} \in L^{r}\left(\mathbb{R}^{d}\right)$ for each compact subset $K \subset \mathbb{R}^{d}$, for which the norm

$$
\|f\|_{W\left(L^{r}, L^{s}\right)}=\left\|F_{f}\right\|_{s}=\|\| f \chi_{Q+x}\left\|_{r}\right\|_{s}
$$

is finite, where $\chi_{K}$ is the characteristic function of $K$ and

$$
F_{f}(x)=\left\|f \chi_{Q+x}\right\|_{r} \in L^{s}\left(\mathbb{R}^{d}\right) .
$$

It is known that if $r_{1} \geq r_{2}$ and $s_{1} \leq s_{2}$ then $W\left(L^{r_{1}}, L^{s_{1}}\right)\left(\mathbb{R}^{d}\right) \subset W\left(L^{r_{2}}, L^{s_{2}}\right)\left(\mathbb{R}^{d}\right)$. If $r=s$ then $W\left(L^{r}, L^{r}\right)\left(\mathbb{R}^{d}\right)=L^{r}\left(\mathbb{R}^{d}\right)$ (see [13-15]).

In this paper, we consider boundedness properties for localization operators acting on Lorentz mixed-normed modulation spaces for the symbols in appropriate function spaces like modulation spaces, Wiener amalgam spaces, and Lorentz spaces with mixed 
norms. Our results extend some results in $[1,12]$ to the Lorentz mixed-normed modulation spaces.

\section{Boundedness of localization operators on Lorentz mixed normed modulation spaces}

We start with the following lemma, which will be used later on.

Lemma 2.1 Let $\frac{1}{P}+\frac{1}{P^{\prime}}=1, \frac{1}{Q_{1}}+\frac{1}{Q_{2}} \geq 1, f \in L\left(P, Q_{1}\right)\left(\mathbb{R}^{2 d}\right), h \in L\left(P^{\prime}, Q_{2}\right)\left(\mathbb{R}^{2 d}\right)$. Then $f * h \in$ $L^{\infty}\left(\mathbb{R}^{2 d}\right)$ and

$$
L\left(P, Q_{1}\right)\left(\mathbb{R}^{2 d}\right) * L\left(P^{\prime}, Q_{2}\right)\left(\mathbb{R}^{2 d}\right) \hookrightarrow L^{\infty}\left(\mathbb{R}^{2 d}\right)
$$

with the norm inequality

$$
\|f * h\|_{\infty} \leq\|f\|_{P Q_{1}}\|h\|_{P^{\prime} Q_{2}},
$$

where $P=\left(p_{1}, p_{2}\right), Q_{1}=\left(Q_{1}^{1}, Q_{1}^{2}\right), Q_{2}=\left(Q_{2}^{1}, Q_{2}^{2}\right)$.

Proof It is well known that there are $L\left(p, q_{1}\right) * L\left(p^{\prime}, q_{2}\right) \hookrightarrow L^{\infty}$ convolution relations between Lorentz spaces and

$$
\|f * h\|_{\infty} \leq\|f\|_{p q_{1}}\|h\|_{p^{\prime} q_{2}}
$$

where $\frac{1}{p}+\frac{1}{p^{\prime}}=1, \frac{1}{q_{1}}+\frac{1}{q_{2}} \geq 1$, by Theorem 3.6 in [5]. Then (2.1) and (2.2) can easily be verified by using iteration and the one variable proofs given in [5].

Let $g \in \mathcal{D}\left(\mathbb{R}^{2 d}\right)$ be a test function such that $\sum_{x \in \mathbb{Z}^{2 d}} T_{x} g \equiv 1$. Let $X\left(\mathbb{R}^{2 d}\right)$ be a translation invariant Banach space of functions with the property that $\mathcal{D} \cdot X \subset X$. In the spirit of [13, 16], the Wiener amalgam space $W(X, L(P, Q))$ with local component $X$ and global component $L(P, Q)$ is defined as the space of all functions or distributions for which the norm

$$
\|f\|_{W(X, L(P, Q))}=\|\| f \cdot T_{\left(z_{1}, z_{2}\right)} \bar{g}\left\|_{X}\right\|_{P Q}
$$

is finite, where $1 \leq P<\infty, 1 \leq Q \leq \infty$. Moreover, different choices of $g \in \mathcal{D}$ yield equivalent norms and give the same space.

The boundedness of $A_{M_{\zeta} a}^{\varphi_{1}, \varphi_{2}}$ for $a \in M^{\infty}$ is established by our next theorem. The proof is similar to Lemma 4.1 in [1] but let us provide the details anyway, for completeness' sake.

\section{Theorem 2.1}

(i) Let $1<P<\infty, 1 \leq Q<\infty$. If $\in M(P, Q)\left(\mathbb{R}^{d}\right)$ and $g \in M^{1}\left(\mathbb{R}^{d}\right)$, then $V_{g} f \in W\left(\mathcal{F} L^{1}, L(P, Q)\right)\left(\mathbb{R}^{2 d}\right)$ with

$$
\left\|V_{g} f\right\|_{W\left(\mathcal{F} L^{1}, L(P, Q)\right)} \leq\|f\|_{M(P, Q)}\|g\|_{M^{1}}
$$

(ii) Let $\frac{1}{P}+\frac{1}{P^{\prime}}=1, \frac{1}{Q_{1}}+\frac{1}{Q_{2}} \geq 1$. If $\in M\left(P, Q_{1}\right)\left(\mathbb{R}^{d}\right)$ and $g \in M\left(P^{\prime}, Q_{2}\right)\left(\mathbb{R}^{d}\right)$, then $V_{g} f \in W\left(\mathcal{F} L^{1}, L^{\infty}\right)\left(\mathbb{R}^{2 d}\right)$ with

$$
\left\|V_{g} f\right\|_{W\left(\mathcal{F} L^{1}, L^{\infty}\right)} \leq\|f\|_{M\left(P, Q_{1}\right)}\|g\|_{M\left(P^{\prime}, Q_{2}\right)} .
$$


Proof (i) Let $\varphi \in \mathcal{S}\left(\mathbb{R}^{d}\right) \backslash\{0\}$ and set $\Phi=V_{\varphi} \varphi \in \mathcal{S}\left(\mathbb{R}^{2 d}\right)$. By using the equality $V_{g} f(x, w)=$ $\left(f \cdot T_{x} \bar{g}\right)^{\wedge}(w)$, we write

$$
\begin{aligned}
\left\|V_{g} f \cdot T_{\left(z_{1}, z_{2}\right)} \bar{\Phi}\right\|_{\mathcal{F} L^{1}} & =\int_{\mathbb{R}^{2 d}}\left|\left(V_{g} f \cdot T_{\left(z_{1}, z_{2}\right)} \bar{\Phi}\right)^{\wedge}(t)\right| d t \\
& =\int_{\mathbb{R}^{2 d}}\left|V_{\Phi} V_{g} f\left(z_{1}, z_{2}, t_{1}, t_{2}\right)\right| d t_{1} d t_{2} \\
& =\int_{\mathbb{R}^{2 d}}\left|V_{\varphi} g\left(-z_{1}-t_{2}, t_{1}\right) V_{\varphi} f\left(-t_{2}, z_{2}+t_{1}\right)\right| d t_{1} d t_{2} \\
& =\int_{\mathbb{R}^{2 d}}\left|V_{\varphi} f\left(u_{1}, u_{2}\right)\right|\left|V_{\varphi} g\left(u_{1}-z_{1}, u_{2}-z_{2}\right)\right| d u_{1} d u_{2} \\
& =\left|V_{\varphi} f\right| *\left|V_{\varphi} g\right|^{\sim}\left(z_{1}, z_{2}\right),
\end{aligned}
$$

for $f, g \in \mathcal{S}\left(\mathbb{R}^{d}\right)$, where $\left(V_{\varphi} g\right)^{\sim}(z)=\left(\overline{V_{\varphi} g}\right)(-z), z \in \mathbb{R}^{2 d}$. Since $f, g \in \mathcal{S}\left(\mathbb{R}^{d}\right)$, then $f \in$ $M(P, Q)\left(\mathbb{R}^{d}\right)$ and $g \in M^{1}\left(\mathbb{R}^{d}\right)$ by Proposition 2 in [8]. So $V_{\varphi} f \in L(P, Q)\left(\mathbb{R}^{2 d}\right)$ and $V_{\varphi} g \in$ $L^{1}\left(\mathbb{R}^{2 d}\right)$. Then, by Proposition 4 in [8], we obtain

$$
\begin{aligned}
\left\|V_{g} f\right\|_{W\left(\mathcal{F} L^{1}, L(P, Q)\right)} & =\|\| V_{g} f \cdot T_{\left(z_{1}, z_{2}\right)} \bar{\Phi}\left\|_{\mathcal{F} L^{1}}\right\|_{P Q} \\
& =\left\|\left|V_{\varphi} f\right| *\left|V_{\varphi} g\right|^{\sim}\right\|_{P Q} \\
& \leq\left\|V_{\varphi} f\right\|_{P Q}\left\|V_{\varphi} g\right\|_{1} \\
& =\|f\|_{M(P, Q)}\|g\|_{M^{1}} .
\end{aligned}
$$

This completes the proof.

(ii) Using Lemma 2.1 and (2.3), we have

$$
\left\|V_{g} f\right\|_{W\left(\mathcal{F} L^{1}, L^{\infty}\right)}=\left\|\left|V_{\varphi} f\right| *\left|V_{\varphi} g\right|^{\sim}\right\|_{\infty} \leq\left\|V_{\varphi} f\right\|_{P Q_{1}}\left\|V_{\varphi} g\right\|_{P^{\prime} Q_{2}}=\|f\|_{M\left(P, Q_{1}\right)}\|g\|_{M\left(P^{\prime}, Q_{2}\right)} .
$$

Theorem 2.2 Let $1<P<\infty, 1 \leq Q<\infty$. If $a \in M^{\infty}\left(\mathbb{R}^{2 d}\right), \varphi_{1}, \varphi_{2} \in M^{1}\left(\mathbb{R}^{d}\right)$, then $A_{M_{\zeta} a}^{\varphi_{1}, \varphi_{2}}$ is bounded on $M(P, Q)\left(\mathbb{R}^{d}\right)$ for every $\zeta \in \mathbb{R}^{2 d}$ with

$$
\left\|A_{M_{\zeta} a}^{\varphi_{1}, \varphi_{2}}\right\|_{B(M(P, Q))} \leq\|a\|_{M^{\infty}}\left\|\varphi_{1}\right\|_{M^{1}}\left\|\varphi_{2}\right\|_{M^{1}}
$$

Proof Let $f \in M(P, Q)\left(\mathbb{R}^{d}\right)$ and $g \in M\left(P^{\prime}, Q^{\prime}\right)\left(\mathbb{R}^{d}\right)$, where $\frac{1}{P}+\frac{1}{P^{\prime}}=1, \frac{1}{Q}+\frac{1}{Q^{\prime}}=1$. Then we write $\overline{V_{\varphi_{1}} f} \in W\left(\mathcal{F} L^{1}, L(P, Q)\right)\left(\mathbb{R}^{2 d}\right)$ and $V_{\varphi_{2}} g \in W\left(\mathcal{F} L^{1}, L\left(P^{\prime}, Q^{\prime}\right)\right)\left(\mathbb{R}^{2 d}\right)$ by above theorem. Moreover, since $M(1,1)\left(\mathbb{R}^{d}\right)=M^{1}\left(\mathbb{R}^{d}\right)$, we have $W\left(\mathcal{F} L^{1}, L^{1}\right)=M^{1}=M(1,1)$ by [16]. Hence using the Hölder inequalities for Wiener amalgam spaces [13] and (2.4) we obtain

$$
\begin{aligned}
\left\|\overline{V_{\varphi_{1}} f} \cdot V_{\varphi_{2}} g\right\|_{M^{1}} & =\left\|\overline{V_{\varphi_{1}} f} \cdot V_{\varphi_{2}} g\right\|_{W\left(\mathcal{F} L^{1}, L^{1}\right)} \\
& \leq\left\|V_{\varphi_{1}} f\right\|_{W\left(\mathcal{F} L^{1}, L(P, Q)\right)}\left\|V_{\varphi_{2}} g\right\|_{W\left(\mathcal{F} L^{1}, L\left(P^{\prime}, Q^{\prime}\right)\right)} \\
& \leq\left\|\varphi_{1}\right\|_{M^{1}}\left\|\varphi_{2}\right\|_{M^{1}}\|f\|_{M(P, Q)}\|g\|_{M\left(P^{\prime}, Q^{\prime}\right) .}
\end{aligned}
$$


Thus by using (2.5) we have

$$
\begin{aligned}
\left|\left\langle A_{M_{\zeta} a}^{\varphi_{1}, \varphi_{2}} f, g\right\rangle\right| & =\left|\left\langle M_{\zeta} a, \overline{V_{\varphi_{1}} f} \cdot V_{\varphi_{2}} g\right\rangle\right| \leq\left\|M_{\zeta} a\right\|_{M(\infty, \infty)}\left\|\overline{V_{\varphi_{1}} f} \cdot V_{\varphi_{2}} g\right\|_{M(1,1)} \\
& \leq\|a\|_{M^{\infty}}\left\|\varphi_{1}\right\|_{M^{1}}\left\|\varphi_{2}\right\|_{M^{1}}\|f\|_{M(P, Q)}\|g\|_{M\left(P^{\prime}, Q^{\prime}\right)}
\end{aligned}
$$

Hence we get

$$
\left\|A_{M_{\zeta} a}^{\varphi_{1}, \varphi_{2}}\right\|_{B(M(P, Q))} \leq\|a\|_{M^{\infty}}\left\|\varphi_{1}\right\|_{M^{1}}\left\|\varphi_{2}\right\|_{M^{1}}
$$

Theorem 2.3 Let $\varphi \in \mathcal{S}\left(\mathbb{R}^{d}\right) \backslash\{0\}$ be a window function. If $1<P, Q<\infty, t^{\prime} \in(1, \infty), s \leq t^{\prime} \leq$ $r$ and $a \in W\left(L^{r}, L^{s}\right)$, then

$$
A_{M_{\zeta} a}^{\varphi, \varphi}: M(t P, t Q)\left(\mathbb{R}^{d}\right) \rightarrow M\left(\left(t P^{\prime}\right)^{\prime},\left(t Q^{\prime}\right)^{\prime}\right)\left(\mathbb{R}^{d}\right)
$$

is bounded for every $\zeta \in \mathbb{R}^{2 d}$, where $\frac{1}{P}+\frac{1}{P^{\prime}}=1, \frac{1}{Q}+\frac{1}{Q^{\prime}}=1$, and $\frac{1}{t}+\frac{1}{t^{\prime}}=1$, and the operator norm satisfies the estimate

$$
\left\|A_{M_{\zeta} a}^{\varphi, \varphi}\right\| \leq\|a\|_{W\left(L^{r}, L^{s}\right)}
$$

Proof Let $t<\infty, f \in M(t P, t Q)\left(\mathbb{R}^{d}\right)$, and $h \in M\left(t P^{\prime}, t Q^{\prime}\right)\left(\mathbb{R}^{d}\right)$. Then we have $V_{\varphi} f \in$ $L(t P, t Q)\left(\mathbb{R}^{2 d}\right)$ and $V_{\varphi} h \in L\left(t P^{\prime}, t Q^{\prime}\right)\left(\mathbb{R}^{2 d}\right)$. Since $V_{\varphi} f \in L(t P, t Q)\left(\mathbb{R}^{2 d}\right)$, then $\left\|V_{\varphi} f\right\|_{(t P)(t Q)}^{*}<$ $\infty$. By using the equality (3.6) in [12], we get

$$
\begin{aligned}
\left\|V_{\varphi} f\right\|_{(t P)(t Q)}^{*} & =\|\| V_{\varphi} f\left\|_{\left(t p_{1}\right)\left(t q_{1}\right)}^{*}\right\|_{\left(t p_{2}\right)\left(t q_{2}\right)}^{*}=\left\|\left(\left\|\left|V_{\varphi} f\right|^{t}\right\|_{p_{1} q_{1}}^{*}\right)^{\frac{1}{t}}\right\|_{\left(t p_{2}\right)\left(t q_{2}\right)}^{*} \\
& =\left(\left\|\left|\left(\left\|\left|V_{\varphi} f\right|^{t}\right\|_{p_{1} q_{1}}^{*}\right)^{\frac{1}{t}}\right|^{t}\right\|_{p_{2} q_{2}}^{*}\right)^{\frac{1}{t}}=\left(\|\|\left|V_{\varphi} f\right|^{t}\left\|_{p_{1} q_{1}}^{*}\right\|_{p_{2} q_{2}}^{*}\right)^{\frac{1}{t}} \\
& =\left(\left\|\left|V_{\varphi} f\right|^{t}\right\|_{P Q}^{*}\right)^{\frac{1}{t}} .
\end{aligned}
$$

Hence we have $\left|V_{\varphi} f\right|^{t} \in L(P, Q)\left(\mathbb{R}^{2 d}\right)$. Similarly, $\left|V_{\varphi} h\right|^{t} \in L\left(P^{\prime}, Q^{\prime}\right)\left(\mathbb{R}^{2 d}\right)$. By the Hölder inequality for Lorentz spaces with mixed norm and (2.6) we have

$$
\begin{aligned}
\left\|V_{\varphi} f \cdot V_{\varphi} h\right\|_{t}^{t} & =\left\|\left|V_{\varphi} f\right|^{t}\left|V_{\varphi} h\right|^{t}\right\|_{1} \leq\left\|\left|V_{\varphi} f\right|^{t}\right\|_{P Q}\left\|\left|V_{\varphi} h\right|^{t}\right\|_{P^{\prime} Q^{\prime}} \\
& =\left\|V_{\varphi} f\right\|_{(t P)(t Q)}^{t}\left\|V_{\varphi} h\right\|_{\left(t P^{\prime}\right)\left(t Q^{\prime}\right)}^{t} .
\end{aligned}
$$

Since $a \in W\left(L^{r}, L^{s}\right)$, then $M_{\zeta} a \in W\left(L^{r}, L^{s}\right)$ for every $\zeta \in \mathbb{R}^{2 d}$. Also since $W\left(L^{r}, L^{s}\right) \subset$ $W\left(L^{t^{\prime}}, L^{t^{\prime}}\right)=L^{t^{\prime}}\left(\mathbb{R}^{2 d}\right)$, then we have

$$
\|a\|_{t^{\prime}}=\left\|M_{\zeta} a\right\|_{t^{\prime}} \leq\left\|M_{\zeta} a\right\|_{W\left(L^{r}, L^{s}\right)}=\|a\|_{W\left(L^{r}, L^{s}\right)} .
$$

By using (2.7), (2.8), and applying again the Hölder inequality, we get

$$
\begin{aligned}
\left|\left\langle A_{M_{\zeta} a}^{\varphi, \varphi} f, h\right\rangle\right| & =\left|\left\langle M_{\zeta} a V_{\varphi} f, V_{\varphi} h\right\rangle\right| \\
& \leq \iint_{\mathbb{R}^{2 d}}\left|M_{\zeta} a(x, w)\right|\left|\left(V_{\varphi} f \cdot V_{\varphi} h\right)(x, w)\right| d x d w \\
& \leq\left\|M_{\zeta} a\right\|_{t^{\prime}}\left\|V_{\varphi} f \cdot V_{\varphi} h\right\|_{t}
\end{aligned}
$$




$$
\begin{aligned}
& \leq\|a\|_{t^{\prime}}\left\|V_{\varphi} f\right\|_{(t P)(t Q)}\left\|V_{\varphi} h\right\|_{\left(t P^{\prime}\right)\left(t Q^{\prime}\right)} \\
& \leq\|a\|_{W\left(L^{r}, L^{s}\right)}\|f\|_{M(t P, t Q)}\|h\|_{M\left(t P^{\prime}, t Q^{\prime}\right)} .
\end{aligned}
$$

If $\left(t p^{\prime}\right)^{\prime},\left(t q^{\prime}\right)^{\prime} \neq \infty$, then $\left(M\left(\left(t P^{\prime}\right)^{\prime},\left(t Q^{\prime}\right)^{\prime}\right)\left(\mathbb{R}^{d}\right)\right)^{*}=M\left(t P^{\prime}, t Q^{\prime}\right)\left(\mathbb{R}^{d}\right)$ by Theorem 8 in [8]. Thus we have from (2.9) that

$$
\left\|A_{M_{\zeta} a}^{\varphi, \varphi} f\right\|_{M\left(\left(t P^{\prime}\right)^{\prime},\left(t Q^{\prime}\right)^{\prime}\right)}=\sup _{0 \neq h \in M\left(t P^{\prime}, t Q^{\prime}\right)} \frac{\left|\left\langle A_{M_{\zeta} a}^{\varphi, \varphi} f, h\right\rangle\right|}{\|h\|_{M\left(t P^{\prime}, t Q^{\prime}\right)}} \leq\|a\|_{W\left(L^{r}, L^{s}\right)}\|f\|_{M(t P, t Q)} .
$$

Hence $A_{M_{\zeta} a}^{\varphi, \varphi}$ is bounded. Also we have

$$
\left\|A_{M_{\zeta} a}^{\varphi, \varphi}\right\|=\sup _{0 \neq f \in M(t P, t Q)} \frac{\left\|A_{M_{\zeta} a}^{\varphi, \varphi} f\right\|_{M\left(\left(t P^{\prime}\right)^{\prime},\left(t Q^{\prime}\right)^{\prime}\right)}}{\|f\|_{M(t P, t Q)}} \leq\|a\|_{W\left(L^{r}, L^{s}\right)}
$$

Theorem 2.4 Let $\varphi \in \bigcap_{1 \leq R, S<\infty} M(R, S)\left(\mathbb{R}^{d}\right)$, where $R=\left(r_{1}, r_{2}\right), S=\left(s_{1}, s_{2}\right)$. If $1 \leq s \leq r \leq \infty$ and $a \in W\left(L^{r}, L^{s}\right)$ then

$$
A_{M_{\zeta} a}^{\varphi, \varphi}: M(P, Q)\left(\mathbb{R}^{d}\right) \rightarrow M(P, Q)\left(\mathbb{R}^{d}\right)
$$

is bounded for every $\zeta \in \mathbb{R}^{2 d}$, with

$$
\left\|A_{M_{\zeta} a}^{\varphi, \varphi}\right\| \leq C\|a\|_{W\left(L^{r}, L^{s}\right)}
$$

for some $C>0$.

Proof Since $a \in W\left(L^{r}, L^{s}\right)$, then $M_{\zeta} a \in W\left(L^{r}, L^{s}\right)$ for every $\zeta \in \mathbb{R}^{2 d}$. Also since $s \leq r$, there exists $1 \leq t_{0} \leq \infty$ such that $s \leq t_{0} \leq r$. Then $W\left(L^{r}, L^{s}\right)\left(\mathbb{R}^{2 d}\right) \subset L^{t_{0}}\left(\mathbb{R}^{2 d}\right)$ and

$$
\left\|M_{\zeta} a\right\|_{t_{0}}=\|a\|_{t_{0}} \leq\|a\|_{W\left(L^{r}, L^{s}\right)}=\left\|M_{\zeta} a\right\|_{W\left(L^{r}, L^{s}\right)}
$$

for all $a \in W\left(L^{r}, L^{s}\right)\left(\mathbb{R}^{2 d}\right)$. Let $B\left(M(P, Q)\left(\mathbb{R}^{d}\right), M(P, Q)\left(\mathbb{R}^{d}\right)\right)$ be the space of the bounded linear operators from $M(P, Q)\left(\mathbb{R}^{d}\right)$ into $M(P, Q)\left(\mathbb{R}^{d}\right)$. Also let $T$ be an operator from $L^{1}\left(\mathbb{R}^{2 d}\right)$ into $B\left(M(P, Q)\left(\mathbb{R}^{d}\right), M(P, Q)\left(\mathbb{R}^{d}\right)\right)$ by $T(a)=A_{M_{\zeta} a}^{\varphi, \varphi}$. Take any $f \in M(P, Q)\left(\mathbb{R}^{d}\right)$ and $h \in M\left(P^{\prime}, Q^{\prime}\right)\left(\mathbb{R}^{d}\right)$. Assume that $a \in W\left(L^{1}, L^{1}\right)\left(\mathbb{R}^{2 d}\right)=L^{1}\left(\mathbb{R}^{2 d}\right)$. By the Hölder inequality we get

$$
\begin{aligned}
|\langle T(a) f, h\rangle|= & \left|\left\langle A_{M_{\zeta}}^{\varphi, \varphi} f, h\right\rangle\right|=\left|\left\langle M_{\zeta} a V_{\varphi} f, V_{\varphi} h\right\rangle\right| \\
\leq & \iint_{\mathbb{R}^{2 d}}\left|M_{\zeta} a(x, w)\right|\left|V_{\varphi} f(x, w) \| V_{\varphi} h(x, w)\right| d x d w \\
= & \iint_{\mathbb{R}^{2 d}}\left|a(x, w)\left\|\left\langle f, M_{w} T_{x} \varphi\right\rangle\right\|\left\langle h, M_{w} T_{x} \varphi\right\rangle\right| d x d w \\
\leq & \iint_{\mathbb{R}^{2 d}}|a(x, w)|\|f\|_{M(P, Q)}\left\|M_{w} T_{x} \varphi\right\|_{M\left(P^{\prime}, Q^{\prime}\right)}\|h\|_{M\left(P^{\prime}, Q^{\prime}\right)} \\
& \times\left\|M_{w} T_{x} \varphi\right\|_{M(P, Q)} d x d w \\
= & \|f\|_{M(P, Q)}\|\varphi\|_{M\left(P^{\prime}, Q^{\prime}\right)}\|h\|_{M\left(P^{\prime}, Q^{\prime}\right)}\|\varphi\|_{M(P, Q)}\|a\|_{1} .
\end{aligned}
$$


Hence by (2.11)

$$
\begin{aligned}
\|T(a) f\|_{M(P, Q)} & =\left\|A_{M_{\zeta} a}^{\varphi, \varphi} f\right\|_{M(P, Q)}=\sup _{0 \neq h \in M\left(P^{\prime}, Q^{\prime}\right)} \frac{\left|\left\langle A_{M_{\zeta} a}^{\varphi, \varphi} f, h\right\rangle\right|}{\|h\|_{M\left(P^{\prime}, Q^{\prime}\right)}} \\
& \leq\|\varphi\|_{M\left(P^{\prime}, Q^{\prime}\right)}\|\varphi\|_{M(P, Q)}\|f\|_{M(P, Q)}\|a\|_{1} .
\end{aligned}
$$

Then

$$
\|T(a)\|=\left\|A_{M_{\zeta} a}^{\varphi, \varphi}\right\|=\sup _{0 \neq f \in M(P, Q)} \frac{\left\|A_{M_{\zeta} a}^{\varphi, \varphi} f\right\|_{M(P, Q)}}{\|f\|_{M(P, Q)}} \leq\|\varphi\|_{M\left(P^{\prime}, Q^{\prime}\right)}\|\varphi\|_{M(P, Q)}\|a\|_{1} .
$$

Thus the operator

$$
T: L^{1}\left(\mathbb{R}^{2 d}\right) \rightarrow B\left(M(P, Q)\left(\mathbb{R}^{d}\right), M(P, Q)\left(\mathbb{R}^{d}\right)\right)
$$

is bounded. Now let $a \in W\left(L^{\infty}, L^{\infty}\right)\left(\mathbb{R}^{2 d}\right)=L^{\infty}\left(\mathbb{R}^{2 d}\right)$. Take any $f \in M(P, Q)\left(\mathbb{R}^{d}\right)$ and $h \in M\left(P^{\prime}, Q^{\prime}\right)\left(\mathbb{R}^{d}\right)$. Then $V_{\varphi} f \in L(P, Q)\left(\mathbb{R}^{2 d}\right), V_{\varphi} h \in L\left(P^{\prime}, Q^{\prime}\right)\left(\mathbb{R}^{2 d}\right)$. Applying the Hölder inequality

$$
\begin{aligned}
|\langle T(a) f, h\rangle| & =\left|\left\langle A_{M_{\zeta} a}^{\varphi, \varphi} f, h\right\rangle\right|=\left|\left\langle M_{\zeta} a V_{\varphi} f, V_{\varphi} h\right\rangle\right| \\
& \leq \iint_{\mathbb{R}^{2 d}}\left|M_{\zeta} a(x, w)\right|\left|V_{\varphi} f(x, w)\right|\left|V_{\varphi} h(x, w)\right| d x d w \\
& \leq\|a\|_{\infty} \iint_{\mathbb{R}^{2 d}}\left|V_{\varphi} f(x, w)\right|\left|V_{\varphi} h(x, w)\right| d x d w \\
& \leq\|a\|_{\infty}\left\|V_{\varphi} f\right\|_{P Q}\left\|V_{\varphi} h\right\|_{P^{\prime} Q^{\prime}} .
\end{aligned}
$$

By using (2.14) we write

$$
\|T(a) f\|_{M(P, Q)}=\left\|A_{M_{\zeta} a}^{\varphi, \varphi} f\right\|_{M(P, Q)}=\sup _{0 \neq h \in M\left(P^{\prime}, Q^{\prime}\right)} \frac{\left|\left\langle A_{M_{\zeta} a}^{\varphi, \varphi} f, h\right\rangle\right|}{\|h\|_{M\left(P^{\prime}, Q^{\prime}\right)}} \leq\|a\|_{\infty}\|f\|_{M(P, Q)} .
$$

Hence by (2.15)

$$
\|T(a)\|=\left\|A_{M_{\zeta} a}^{\varphi, \varphi}\right\|=\sup _{0 \neq f \in M(P, Q)} \frac{\left\|A_{M_{\zeta} a}^{\varphi, \varphi} f\right\|_{M(P, Q)}}{\|f\|_{M(P, Q)}} \leq\|a\|_{\infty} .
$$

That means the operator

$$
T: L^{\infty}\left(\mathbb{R}^{2 d}\right) \rightarrow B\left(M(P, Q)\left(\mathbb{R}^{d}\right), M(P, Q)\left(\mathbb{R}^{d}\right)\right)
$$

is bounded. Combining (2.13) and (2.16) we obtain

$$
T: L^{t}\left(\mathbb{R}^{2 d}\right) \rightarrow B\left(M(P, Q)\left(\mathbb{R}^{d}\right), M(P, Q)\left(\mathbb{R}^{d}\right)\right)
$$

is bounded by interpolation theorem for $1 \leq t \leq \infty$. That means the localization operator

$$
A_{M_{\zeta} a}^{\varphi, \varphi}: M(P, Q)\left(\mathbb{R}^{d}\right) \rightarrow M(P, Q)\left(\mathbb{R}^{d}\right)
$$


is bounded for $1 \leq t \leq \infty$. Hence there exists $C>0$ such that

$$
\|T(a)\|=\left\|A_{M_{\zeta} a}^{\varphi, \varphi}\right\| \leq C\|a\|_{t}
$$

This implies that it is also true for $1 \leq t_{0} \leq \infty$. From (2.10) and (2.17) we write

$$
\|T(a)\|=\left\|A_{M_{\zeta} a}^{\varphi, \varphi}\right\| \leq C\|a\|_{t_{0}} \leq C\|a\|_{W\left(L^{r}, L^{s}\right)} .
$$

Proposition 2.1 Let $\varphi \in \bigcap_{1 \leq R, S<\infty} M(R, S)\left(\mathbb{R}^{d}\right)$, where $R=\left(r_{1}, r_{2}\right), S=\left(s_{1}, s_{2}\right)$. If $0<s \leq 1$ and $a \in W\left(L^{1}, L^{s}\right)\left(\mathbb{R}^{2 d}\right)$ then

$$
A_{M_{\zeta} a}^{\varphi, \varphi}: M(P, Q)\left(\mathbb{R}^{d}\right) \rightarrow M(P, Q)\left(\mathbb{R}^{d}\right)
$$

is bounded.

Proof Let $0<s \leq 1$ and let $a \in W\left(L^{1}, L^{s}\right)\left(\mathbb{R}^{2 d}\right)$. Then $M_{\zeta} a \in W\left(L^{1}, L^{s}\right)$ for every $\zeta \in$ $\mathbb{R}^{2 d}$. Since $W\left(L^{1}, L^{s}\right)\left(\mathbb{R}^{2 d}\right) \subset L^{1}\left(\mathbb{R}^{2 d}\right)$, there exists a number $C>0$ such that $\left\|M_{\zeta} a\right\|_{1} \leq$ $C\left\|M_{\zeta} a\right\|_{W\left(L^{1}, L^{s}\right)}$. Hence by $(2.12)$,

$$
\begin{aligned}
\left\|A_{M_{\zeta} a}^{\varphi, \varphi}\right\| & \leq\|\varphi\|_{M\left(P^{\prime}, Q^{\prime}\right)}\|\varphi\|_{M(P, Q)}\left\|M_{\zeta} a\right\|_{1} \\
& \leq C\|\varphi\|_{M\left(P^{\prime}, Q^{\prime}\right)}\|\varphi\|_{M(P, Q)}\left\|M_{\zeta} a\right\|_{W\left(L^{1}, L^{s}\right)} \\
& =C\|\varphi\|_{M\left(P^{\prime}, Q^{\prime}\right)}\|\varphi\|_{M(P, Q)}\|a\|_{W\left(L^{1}, L^{s}\right)} .
\end{aligned}
$$

Then the localization operator from $M(P, Q)\left(\mathbb{R}^{d}\right)$ into $M(P, Q)\left(\mathbb{R}^{d}\right)$ is bounded for $0<s \leq 1$.

Proposition 2.2 Let $\varphi \in \bigcap_{1 \leq R, S<\infty} M(R, S)\left(\mathbb{R}^{d}\right)$, where $R=\left(r_{1}, r_{2}\right), S=\left(s_{1}, s_{2}\right)$. If $1 \leq P, Q<$ $\infty$ and $a \in L\left(P^{\prime}, Q^{\prime}\right)\left(\mathbb{R}^{2 d}\right)$ then the localization operator

$$
A_{M_{\zeta} a}^{\varphi, \varphi}: M(P, Q)\left(\mathbb{R}^{d}\right) \rightarrow M(P, Q)\left(\mathbb{R}^{d}\right)
$$

is bounded, where $\frac{1}{P}+\frac{1}{P^{\prime}}=1, \frac{1}{Q}+\frac{1}{Q^{\prime}}=1$.

Proof Let $a \in L\left(P^{\prime}, Q^{\prime}\right)\left(\mathbb{R}^{2 d}\right)$. Then $M_{\zeta} a \in L\left(P^{\prime}, Q^{\prime}\right)\left(\mathbb{R}^{2 d}\right)$ for every $\zeta \in \mathbb{R}^{2 d}$ with $\left\|M_{\zeta} a\right\|_{P^{\prime} Q^{\prime}}=\|a\|_{P^{\prime} Q^{\prime}}$. Take any $f \in M(P, Q)\left(\mathbb{R}^{d}\right)$ and $h \in M\left(P^{\prime}, Q^{\prime}\right)\left(\mathbb{R}^{d}\right)$. Applying the Hölder inequality we have by (2.11)

$$
\begin{aligned}
\left|\left\langle A_{M_{\zeta}}^{\varphi, \varphi} f, h\right\rangle\right| & \leq \iint_{\mathbb{R}^{2 d}}\left|M_{\zeta} a(x, w)\left\|V_{\varphi} f(x, w)\right\|\left\langle h, M_{w} T_{x} \varphi\right\rangle\right| d x d w \\
& \leq \iint_{\mathbb{R}^{2 d}}\left|a(x, w)\left\|V_{\varphi} f(x, w) \mid\right\| h\left\|_{M\left(P^{\prime}, Q^{\prime}\right)}\right\| M_{w} T_{x} \varphi \|_{M(P, Q)} d x d w\right. \\
& =\|h\|_{M\left(P^{\prime}, Q^{\prime}\right)}\|\varphi\|_{M(P, Q)} \iint_{\mathbb{R}^{2 d}}\left|a(x, w) \| V_{\varphi} f(x, w)\right| d x d w \\
& \leq\|h\|_{M\left(P^{\prime}, Q^{\prime}\right)}\|\varphi\|_{M(P, Q)}\|f\|_{M(P, Q)}\|a\|_{P^{\prime} Q^{\prime}} .
\end{aligned}
$$


Similarly to (2.12), we get

$$
\left\|A_{M_{\zeta} a}^{\varphi, \varphi}\right\| \leq\|\varphi\|_{M(P, Q)}\|a\|_{P^{\prime} Q^{\prime}}
$$

Then the localization operator $A_{M_{\zeta} a}^{\varphi, \varphi}$ from $M(P, Q)\left(\mathbb{R}^{d}\right)$ into $M(P, Q)\left(\mathbb{R}^{d}\right)$ is bounded.

Corollary 2.1 It is known by Proposition 2 in [8] that $\mathcal{S}\left(\mathbb{R}^{d}\right) \subset M(R, S)\left(\mathbb{R}^{d}\right)$ for $1 \leq R, S<$ $\infty$. Then $\mathcal{S}\left(\mathbb{R}^{d}\right) \subset \bigcap_{1 \leq R, S<\infty} M(R, S)\left(\mathbb{R}^{d}\right)$. So, Theorem 2.4, Propositions 2.1 and 2.2 are still true under the same hypotheses for them if $\varphi \in \mathcal{S}\left(\mathbb{R}^{d}\right)$.

Corollary 2.2 It is known [8] that if $P=p$ and $Q=q$, then Lorentz mixed-normed modulation space $M(P, Q)\left(\mathbb{R}^{d}\right)$ is the Lorentz type modulation space $M(p, q)\left(\mathbb{R}^{d}\right)$. Therefore our theorems hold for a Lorentz type modulation space rather than for a Lorentz mixed-normed modulation space.

\section{Competing interests}

The author declares that she has no competing interests.

Received: 21 August 2014 Accepted: 24 October 2014 Published: 31 Oct 2014

\section{References}

1. Cordero, E, Gröchenig, K: Time-frequency analysis of localization operators. J. Funct. Anal. 205(1), 107-131 (2003)

2. Gröchenig, K: Foundations of Time-Frequency Analysis. Birkhäuser, Boston (2001)

3. Feichtinger, HG: Modulation spaces on locally compact Abelian groups. Technical Report, University of Vienna (1983)

4. Hunt, RA: On $L(p, q)$ spaces. Enseign. Math. 12(4), 249-276 (1966)

5. O'Neil, R: Convolution operators and $L(p, q)$ spaces. Duke Math. J. 30, 129-142 (1963)

6. Blozinski, AP: Multivariate rearrangements and Banach function spaces with mixed norms. Trans. Am. Math. Soc. 1, 149-167 (1981)

7. Fernandez, DL: Lorentz spaces, with mixed norms. J. Funct. Anal. 25, 128-146 (1977)

8. Sandıkçı, A: On Lorentz mixed normed modulation spaces. J. Pseud.-Differ. Oper. Appl. 3, 263-281 (2012)

9. Sandıkçı, A: Continuity of Wigner-type operators on Lorentz spaces and Lorentz mixed normed modulation spaces. Turk. J. Math. 38, 728-745 (2014). doi:10.3906/mat-1311-43

10. Gürkanli, AT: Time-frequency analysis and multipliers of the spaces $M(p, q)\left(R^{d}\right)$ and $S(p, q)\left(R^{d}\right)$. J. Math. Kyoto Univ. 46(3), 595-616 (2006)

11. Sandıkçı, A, Gürkanlı, AT: Gabor analysis of the spaces $M(p, q, w)\left(\mathbb{R}^{d}\right)$ and $S(p, q, r, w, \omega)\left(\mathbb{R}^{d}\right)$. Acta Math. Sci. 31(1), 141-158 (2011)

12. Sandıkçı, A, Gürkanlı, AT: Generalized Sobolev-Shubin spaces, boundedness and Schatten class properties of Toeplitz operators. Turk. J. Math. 37, 676-692 (2013). doi:10.3906/mat-1203-5

13. Feichtinger, HG: Banach convolution algebras of Wiener type. In: Proc. Conf. on Functions, Series, Operators (Budapest 1980). Colloq. Math. Soc. Janos Bolyai, vol. 35, pp. 509-524. North Holland, Amsterdam (1983)

14. Heil, C: An introduction to weighted Wiener amalgams. In: Krishna, M, Radha, R, Thangavelu, S (eds.) Wavelets and Their Applications (Chennai, January 2002), pp. 183-216. Allied Publishers, New Delhi (2003)

15. Holland, F: Harmonic analysis on amalgams of $L^{p}$ and $\ell^{q}$. J. Lond. Math. Soc. 10(2), 295-305 (1975)

16. Feichtinger, HG, Luef, F: Wiener amalgam spaces for the fundamental identity of Gabor analysis. Collect. Math. 57, Vol. Extra, 233-253 (2006)

10.1186/1029-242X-2014-430

Cite this article as: Sandıkçı: Boundedness of localization operators on Lorentz mixed-normed modulation spaces. Journal of Inequalities and Applications 2014, 2014:430 